Research highlights:

- Peak demand is shaved for photovoltaic battery systems with electric vehicles.

- A central planner's objective is applied on a decentral operator's system.

- Uncertainties from load demand, photovoltaic, and electric vehicle are considered.

- A battery reserve capacity and the relaxation of the demand limit are implemented.

- A reduction of the daily peak by $17 \%$ to $52 \%$ is achieved. 


\title{
Unit Commitment of Photovoltaic-Battery Systems: An Advanced Approach Considering Uncertainties from Load, Electric Vehicles, and Photovoltaic
}

\author{
Uwe Langenmayr ${ }^{\mathrm{a}, *}$, Weimin Wang ${ }^{\mathrm{b}}$, Patrick Jochem ${ }^{\mathrm{a}, \mathrm{c}}$ \\ ${ }^{a}$ Institute for Industrial Production (IIP) at Karlsruhe Institute of Technology (KIT), Kaiserstr. 12, 76131 Karlsruhe, Germany \\ ${ }^{b}$ Department of Engineering Technology, University of North Carolina at Charlotte, Charlotte, NC 28223, US \\ ${ }^{c}$ Department of Energy Systems Analysis, Institute of Engineering Thermodynamics, German Aerospace Center (DLR), Pfaffenwaldring \\ 38-40, 70569 Stuttgart, Germany
}

\begin{abstract}
Increasing use of renewable energy leads to change in load flows from predictable generation and inelastic demand to more volatile and price-elastic patterns, especially on the distribution level. New applications such as electric vehicles further increase the demand of electricity. Therefore, a reliable, local control of load flexibilities is a key competence of future system operators. This paper presents a central planner - decentral operator approach to schedule local electricity flows. The central planner conducts a two-stage optimization to derive the demand limit and a corresponding battery schedule, while the decentral operator simply applies the battery schedule and heuristically reacts to unforeseen deviations between the forecasted and actual loads and power generation. Privacy concerns of the decentral planner are avoided as no private information is shared with the central planner. A relaxation factor and a reserve capacity for the battery are derived from a Monte Carlo simulation to consider the underlying uncertainties of load, photovoltaic generation, and electric vehicle charging. Our results show that the load of the decentral operator can be limited reliably for six days of the considered week and a maximum reduction of $2.6 \mathrm{~kW}(52 \%)$ of peakload has been accomplished. Furthermore, the approach is suitable for systems with limited computational resources at the place of the decentral operator, which is the common case in this field.
\end{abstract}

Keywords: PV-battery systems, Peak shaving, Uncertainty, Monte Carlo simulation, Electric vehicle, Optimization

\section{Nomenclature}

Abbreviations
DCM $\quad$ Demand charge management
$E V \quad$ Electric vehicle
$P V \quad$ Photovoltaic
Greek Symbols
$\alpha \quad$ Relaxation factor
Subscripts
$f \quad$ Forecasted values
$n \quad$ Quarter-hour timestep
$t \quad$ 1-minute timestep

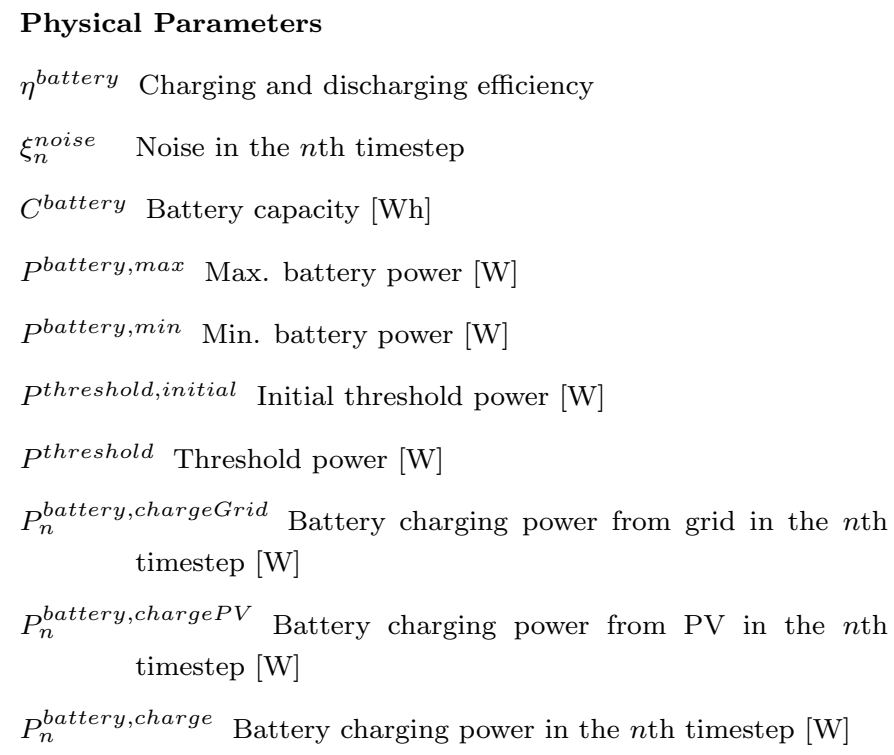

${ }^{*}$ Corresponding author at: Institute for Industrial Production (IIP) at Karlsruhe Institute of Technology (KIT), Hertzstr. 16, D-76187 Karlsruhe, Germany.

Email address: uwe.langenmayr@kit.edu (Uwe Langenmayr) 


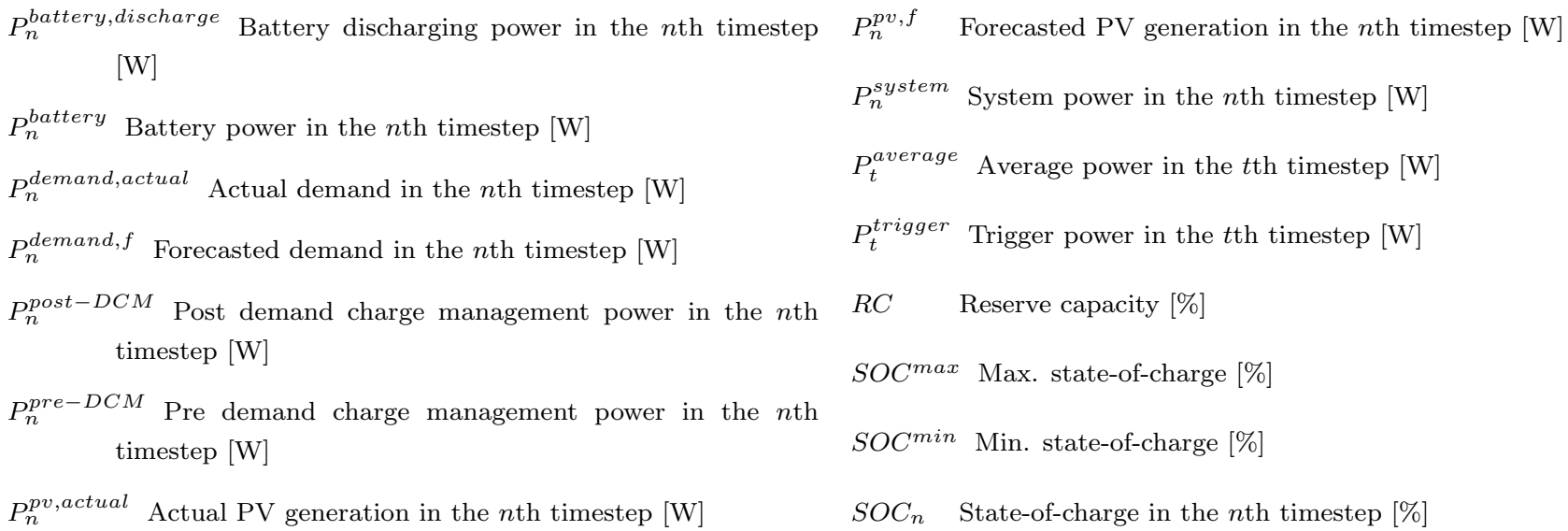

\section{Introduction}

Decreasing price for photovoltaic (PV) and battery systems in combination with efforts to mitigate greenhouse gas emissions for electricity generation is leading to a decentralization in energy systems (Alanne and Saari, 2006). This includes electricity generation (mainly by PV) and the provision of flexibilities (e.g., by battery systems). Thus, load flows are changing considerably from predictable and inelastic to more volatile and price-elastic patterns (Kaschub et al., 2016), which may influence grid stability. Hence, a reliable, local control of load flexibilities is a key competence of future system operators. If these control mechanisms are applied in real applications, they should be able to account for important parameters (such as PV and load prognosis) including their uncertainties, and to use stable (i.e., noncomplex) control mechanisms in order to assure a reliable operation of the electricity system. These decentralized but still highly reliable electricity systems are a cornerstone of the energy transition and, consequently, developing reliable control algorithms for controlling these decentral systems is a core request for the scientific community.

There has already been extensive research on distributed PV-battery systems. Some research intends to develop energy dispatch strategies based on given component sizes or PV generation profiles. The energy dispatch strategy can be proposed from the perspectives of demand side management (Matallanas et al. (2012), Di Giorgio and Pimpinella (2012)), battery storage scheduling (Farah et al. (2016), Chua et al. (2015)), heat device scheduling (Jochem et al. (2015), Dengiz and Jochem (2020)), electric vehicle (EV) charging scheduling (Seddig et al., 2019), and the combination of both active demand side and storage management (Paterakis et al., 2016) or privacy issues (Buchmann et al., 2013). Energy dispatch can be optimized for different objectives, such as operational cost (Ranaweera and Midtgård (2016), Klingler and Teichtmann (2017), Zhong et al. (2016)), self-consumption (Castillo-Cagigal et al., 2011), self-sufficiency (Li et al., 2018), power smoothing (Arcos-Aviles et al. (2017), Wang et al. (2020)), and peak demand shaving (Lu et al., 2018). In addition, multiple objectives can be considered simultaneously when developing optimal energy dispatch of PV-battery systems.

\subsection{Energy Dispatch for Peak Demand Reduction}

Peak demand shaving can be performed at different levels: single buildings, communities, distribution circuit levels and even larger areas. Only peak demand reduction for a single building is reviewed below with recognition of the existence of studies on shaving peak demand for more than a single building (Luthander et al. (2016), Lopes et al. (2016), Reihani et al. (2016)).

Some studies simply assume the forecasts are accurately known in advance, leading to the formulation of an offline predictive optimization problem. Riffonneau et al. (2011) use dynamic programming to minimize the operating cost of a grid-connected PV system with energy storage. The peak demand is treated as a constraint by specifying an upper limit for the power exchange with the grid. They conclude that the approach achieves good results under simulated conditions, but the results under real conditions depend strongly on the forecast accuracy. Erdinc (2014) formulates 
a mixed integer linear programming model to minimize the total daily cost of electricity consumption under different demand response strategies, one of which is peak power limiting. Ratnam et al. (2015) optimize the battery's dayahead schedule via a quadratic programming-based algorithm. The problem is formulated to balance two objectives: minimizing the impact of residential PV systems on the grid (i.e., the alleviation of peak demand and reverse power flow) and increasing the operational savings to house owners. The two objectives are balanced through a user-specified weighting matrix in the cost function. The approach resulted in the battery to discharge mainly during shoulder and peak periods, leading to a peak demand reduction.

Due to the intrinsic volatility of PV power generation and electric loads, the optimal control of PV-battery systems needs to consider the deviation between predicted and actual power profiles in empirical applications. In this regard, many studies have adopted a hierarchical control strategy. At the upper level, a predictive optimization is made based on the forecasted load profile, PV generation profile, and electricity prices. This predictive optimization generates the schedule of power management for a fixed time window (e.g., 24 hours). At the lower level, the dispatch schedule from the upper level is adjusted online to accommodate the prediction error. As the time window used for the lower level control recedes, the upper level control is updated. Along this thread of thought, Wang et al. (2014) propose a two-stage control strategy to minimize the household owner's utility cost, including both energy cost and peak demand cost. The two-stage control consists of a global control tier and a local control tier. The global control tier conducts a convex optimization to plan the future charging/discharging schedule for a billing period of one month. The local control tier refines the storage control policy determined from the global tier dynamically in response to the difference between predicted and actual power generation and consumption profiles. Compared to three heuristicbased operation strategies, the two-stage approach reduces the owner's electricity bill while indirectly reducing peak demand. A similar approach is used by Hanna et al. (2014) and serves as the basis of our approach. In their work, a linear optimization model is used at midnight to set the initial daily peak demand, called "load demand target". The inputs to the linear optimization include the day-ahead load and PV forecasts, the initial battery charge state, and the physical constraints of the PV-battery system. To cope with the forecast error, Hanna et al. adopt two measures. First, the linear optimization model is rerun hourly by replacing forecasted load and PV power with known data for past hours, which may lead to an updated load target. Second, a real-time operation algorithm, termed "trigger", is used within every 15-min interval to maintain the load demand target by responding to load and PV fluctuations at 1-minute resolution. When the original load target is jeopardized, the trigger resorts to battery discharge if the battery has available capacity and the discharging power satisfies the battery operational constraint. Based on the simulation for a university building in California, Hanna et al. have shown that with their proposed approach, the battery storage reduces peak demand by about $6 \%$ in summer and about $9.3 \%$ in winter. However, over forecasting PV power generation or under forecasting load may severely compromise the peak demand reduction capability.

As discussed above, the real-time operation applied at the lower level of the hierarchical control is one possible approach to deal with imperfect forecasting when developing optimal energy dispatch in PV-battery systems. Another approach is to quantify the forecasting uncertainty and then apply stochastic optimization for energy dispatch. However, because research papers focusing on real-time peak demand shaving while accounting for uncertainties are rarely found in literature, an overview of studies considering the uncertainty of PV-battery system components in general are provided in next subsection.

\subsection{PV-Battery Systems Considering Uncertainties}

The uncertainties are sourced from different components of PV-battery systems. Weather factors (e.g., clouds and ambient air temperature) contribute to uncertainties of PV power generation. In addition to weather factors, occupancy behavior is another important source of electric load uncertainty. The uncertainties associated with EVs come from vehicle availability for charging (i.e., plug-in times) and their charging needs (i.e., energy demand). If real-time pricing is used, the supply and demand fluctuations based on these (uncertain) price changes cause another uncertainty. 
Hemmati and Saboori (2017) combine a stochastic mixed-integer non-linear programming and Monte Carlo simulation to address the uncertain PV generation of a residential PV-battery system. The PV power uncertainty is captured by using Gaussian probability density functions for solar radiation and ambient temperature. The stochastic programming aims to determine an optimal battery capacity and a charge/discharge regime through annualized total cost optimization. Correa-Florez et al. (2018) minimize the expected day-ahead operation cost, which includes energy procurement cost and battery cycling cost, of a home energy management system through optimal scheduling the battery unit with a two-stage mixed integer non-linear programming. They consider both load and PV uncertainties using probabilistic forecasts and the resulted generation of nine scenarios. Appino et al. (2018) also rely on probabilistic forecasts for the aggregated PV power generation and loads to compute a dispatch schedule. Their objective is to minimize the residential building electricity cost, using time-varying cost coefficients in a quadratic cost function. Schwarz et al. (2018) use Markov Chains to consider the uncertainties of load demand, PV generation and electricity price. Load demand, PV generation, and electricity price profiles are generated by considering the transition probabilities of weather occurrences (e.g., cloudiness and ambient temperature) and their interactions.

Uncertainties from EV loads are typically modeled with probability distribution functions or Markov processes. When maximizing the utilization of PV generation through smart charging strategies of EV fleets in a parking garage, Seddig et al. (2019) consider the EV charging uncertainty from three aspects: arrival time, departure time, and trip distance, the stochastics of all of which are described with probability distribution functions. Thomas et al. (2018) present a stochastic mixed-integer linear programming model for a commercial PV-battery system with an EV fleet. The EV stochastics are due to the arrival time, the detention time for EV charging, and the initial state of charge of EV battery, all of which are described with different probability distribution functions. Thomas et al. conclude that the stochastic approach performs better in terms of the total expected daily cost of the system than the deterministic approach. Wang et al. (2020) aim to schedule EV charging connected to one charging infrastructure in a centralized way. A stochastic linear programming model is used to address the problem of charging management. The approach includes a Markov Chain to handle the EV uncertainty of the arriving time, the departure time, and the energy demand. The approach leads to a peak demand reduction and a more equally distributed charging of the EV during the day, compared to a non-controlled charging.

The literature review has identified many studies on optimal energy dispatch of grid-connected PV battery systems. However, the work considering uncertainty towards peak demand limitation is missing, especially for residential households with PV, battery storage, and an EV. Consequently, our contribution to the literature is to develop a reliable, privacy-compliant, and real-time ready control algorithm, which can cope with fluctuating decentral electricity generation (e.g., PV) and load flexibilities (provided by a battery) as well as the underlying uncertainties from PV, load, and EV. In this vein, we are answering the following three research questions in our contribution:

- Is our approach a convincing tool in terms of allowing a central planner (i.e., grid operator) to make use of the load flexibilities of decentral customers without knowing private information and without neglecting uncertainties from PV, load, and EV?

- Is it possible for a decentral operator to follow the battery schedule proposed by the central planner without requiring high calculation capacities, allowing a reliable, empirical application?

- Which impact has this approach on the residual load of the decentral application and how do the developed mechanisms tackle the uncertainty from PV generation, electric load, and EV demand?

For answering these research questions, we propose the following structure of the paper: Section 2 outlines the central planner - decentral operator approach and the PV battery system, which is followed by Section 3 on the discussion of the optimization framework and the underlying procedure. Section 4 provides input data, forecasting uncertainty modeling and the Monte Carlo simulation for the derivation of reserve capacity and relaxation factor. 
Section 5 dwells on the results of the approach by analyzing the peak demand reduction and the impact of the uncertainty consideration. The last section provides a short conclusion and further outlook on future research.

\section{Research Design}

Our research design focuses on a central planner - decentral operator approach to cope with privacy (cf. Section 2.1). The decentral planer disposes a residential household endowed with PV, a battery, and an EV (cf. Section 2.2). Our unit commitment approach, extended from Hanna et al. (2014), is outlined in Section 2.3.

\subsection{Central Planner and Decentral Operator}

In the central planner - decentral operator approach, the task of the central planner is to support the decentral operator to limit its daily peak demand. On a daily basis, the central planner derives an optimal operating reserve using the battery energy, called reserve capacity, and an optimal peak demand limit, called threshold power, and provides this information to the decentral operator. Furthermore, the central planner provides a battery schedule to the decentral operator, which is optimized to support the peak demand limitation. On the other hand, the decentral operator's task is to apply the battery schedule of the central planner.

The reserve capacity, the threshold power, and the battery schedule are based on information of the decentral operator. Therefore, the decentral operator provides the necessary information to the central planner. The information including the total annual electricity demand, the PV generation capacity, the EV, and the EV charging station is used by the central planner to forecast the load, PV generation, and EV demand of the decentral operator. The information regarding battery capacity and the current state-of-charge (SOC) of the battery is used to schedule battery operation. In order to adjust the battery schedule regarding deviations between forecasted profiles and occurred profiles, the highest residual peak demand of the past hours of the day is tracked. For privacy reasons, the detailed information of the load profile is not shared. The schematic central planner - decentral operator approach is depicted in Figure 1.

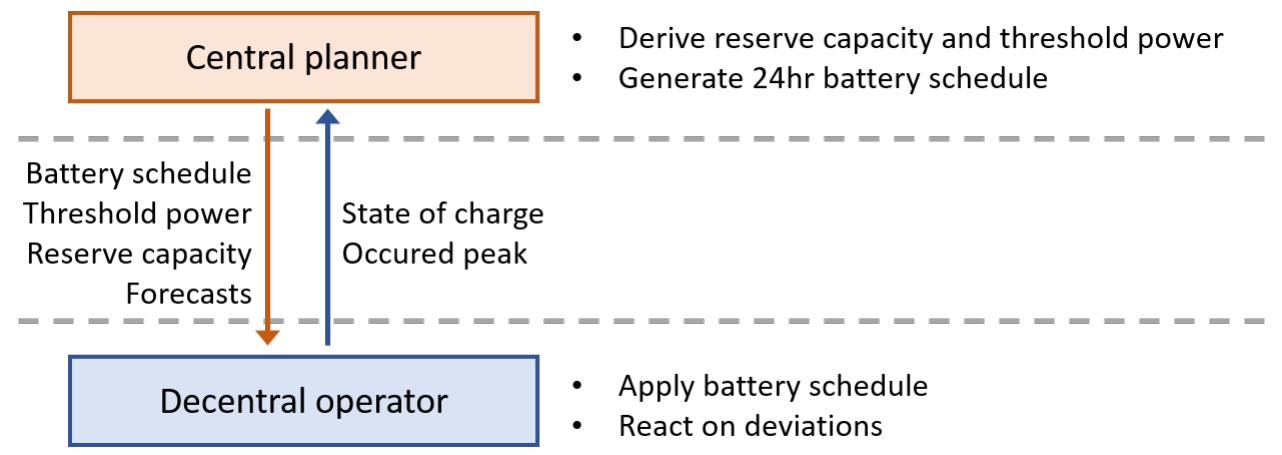

Figure 1: Scheme of the tasks and the exchanged data of the central planner and decentral operator.

\subsection{System's Characteristics of the Decentral Operator}

In this work, the central planner is assumed to be a grid operator whereas the decentral operator is assumed to be a residential homeowner. The PV-battery system is a grid-connected and AC-coupled system, which consists of PV modules, inverters, a Lithium-ion battery, a single EV, an EV charging station, and other electrical accessories.

The battery is assumed to have an equal charging and discharging efficiency, which includes the losses of power conditioning equipment associated with the battery. Battery capacity degradation due to time and charging cycles is not considered. Although with the simplifications mentioned above, the battery is operated in a component-friendly manner, taking into account appropriate charging and discharging power limits and SOC boundaries. For this gridconnected system, battery charging from the grid is allowed, but discharging into the grid is not allowed. Figure 2 shows the schematic of the considered PV-battery system. 


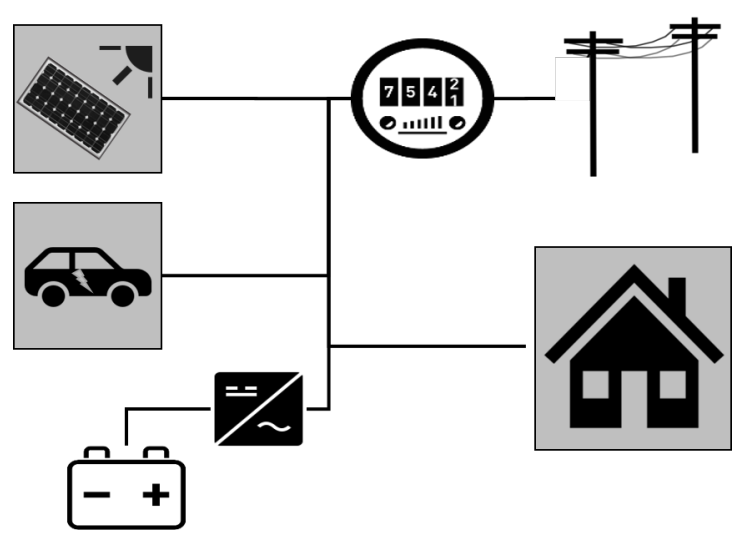

Figure 2: Schematic of the decentral operator's residential PV-battery system with EV.

\subsection{An Outline of our Unit Commitment Approach}

The approach is based on a running window approach and involves the central planner's and decentral operator's tasks. Every day, at midnight, the central planner conducts the first stage of a two-stage optimization to derive the demand limit, called threshold power, which sets the upper limit of the residual load of the decentral operator. This threshold power is used directly in the second stage of the two-stage optimization. The aim of this optimization is to derive a 24-hour battery schedule for the decentral operator's battery, which satisfies the threshold power derived in the first-stage optimization. The forecasts, the battery schedule, and the threshold power are transferred to the decentral operator's system. Here, the decentral operator system applies the battery schedule for the upcoming hour. In addition, the decentral operator's task is to react on deviations between the forecasts and measured data from the system. If these deviations jeopardize the threshold power, the system reschedules the battery to avoid exceeding the threshold power using the trigger function (similar to Hanna et al. (2014)). The trigger function is a mechanism, which measures the data in real-time and implements additional discharge of the battery, if the demand limit adherence is jeopardized. After the hour is covered by the unit commitment on the decentral operator level, the information regarding the occurred peak of the past hour and the SOC of the battery at the end of the hour is exchanged with the central planner. The central planner conducts the second-stage optimization again to schedule the battery for the next remaining hours of the day. This continues until midnight of the next day. The central planner conducts the first-stage optimization again and continues the process until the week is covered. A simplified depiction of the process is shown in Figure 3. The following Section 3 will present the used methodology in detail. 


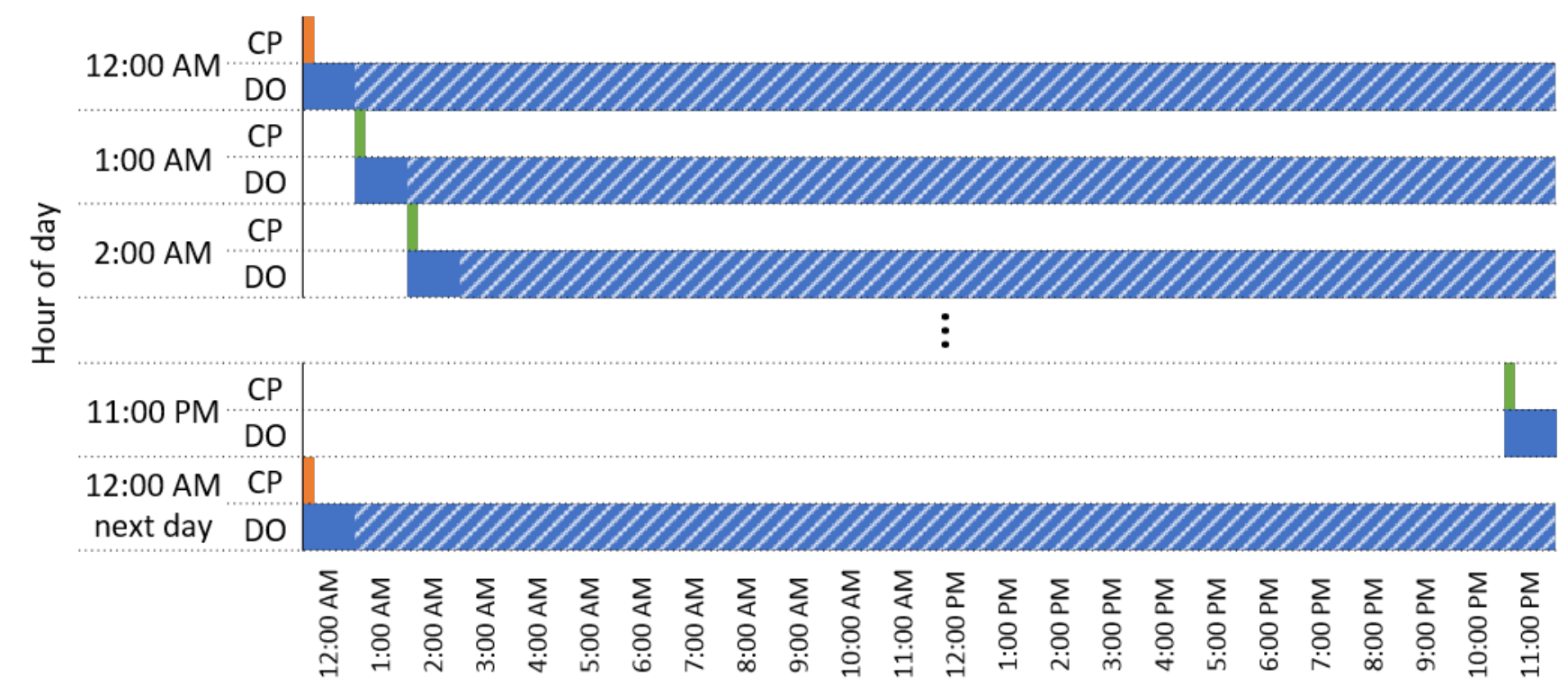

Considered time period for battery schedule calculation

\begin{tabular}{|c|c|c|c|}
\hline $\begin{array}{c}\text { Calculating threshold power } \\
\text { and battery schedule }\end{array}$ & $\begin{array}{c}\text { Updating battery } \\
\text { schedule }\end{array}$ & $\begin{array}{c}\text { Applying battery } \\
\text { schedule }\end{array}$ & $\begin{array}{c}\text { Calculated but not } \\
\text { applied battery schedule }\end{array}$ \\
\hline
\end{tabular}

Figure 3: Implemented running window approach. At $12 \mathrm{AM}$, the central planner (CP) conducts the two-stage optimization (orange block) and transfers the derived threshold power and the battery schedule to the decentral operator (DO). Though the DO has the schedule for 24 hours (full blue block), it only applies it for the first hour (solid blue block). After the application, the DO sends the occurred residual peak load and the updated SOC to the CP, who conducts the second-stage of the two stage optimization again (green block) and sends the battery schedule to the DO. This continues until the last hour of the day was covered. Afterwards, at 12 AM of the next day, the process starts again on the CP level with the two-stage optimization.

\section{Methodology}

The methodology starts with a two-stage optimization by the central planner and continues with the battery dispatch schedule application by the decentral operator.

\subsection{Two-stage Optimization by the Central Planner}

The central planner uses a two-stage optimization to derive the optimal battery schedule for the decentral operator. The first stage computes the minimal reduction level, which is further relaxed to consider the underlying uncertainties, because actual profiles may deviate from forecasts. The relaxed reduction level is the threshold power, which is treated as the residual load limit in the second-stage optimization to obtain the battery schedule. The process is depicted in Figure 4 and explained further in the following two subsections. 


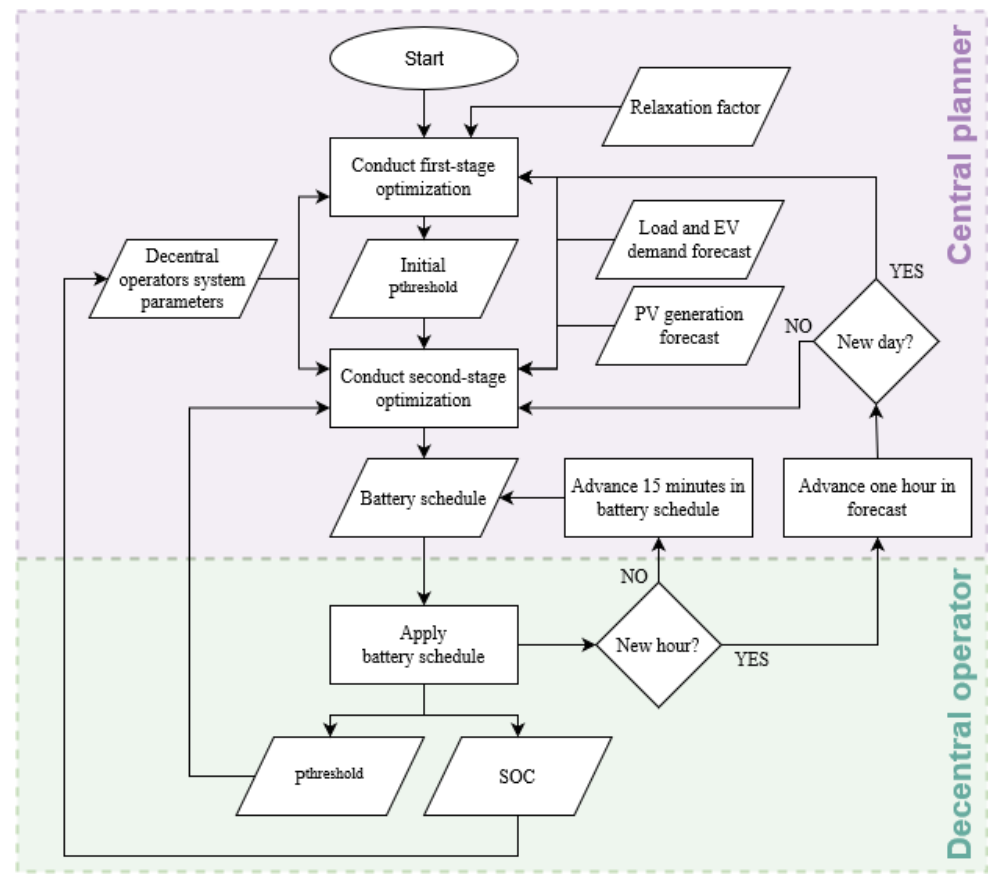

Figure 4: Approach from the central planner point of view.

\subsubsection{First-stage Optimization: Threshold Power Determination}

Equation 1 represents the objective function, which takes a quadratic programming form to minimize the square of the difference between the forecasted demand $P_{n}^{\text {demand,f }}$ and the system's output $P_{n}^{\text {system }}$ during critical quarterhour steps. Critical quarter-hour steps are those timesteps, when the forecasted demand $P_{n}^{\text {demand,f }}$ is larger than the forecasted PV generation $P_{n}^{p v, f}$ and, therefore, resulting in a positive residual load (Hanna et al., 2014).

$$
\begin{array}{r}
\min f\left(P_{n}^{\text {demand }, f}, P_{n}^{\text {system }}\right)=\sum_{n=1}^{N}\left(P_{n}^{\text {demand }, f}-P_{n}^{\text {system }}\right)^{2} \\
\text { with } P_{n}^{\text {demand }, f}>P_{n}^{\text {pv,f }}
\end{array}
$$

for all $n \in N$.

The quadratic program is solved at the beginning of each day and reduces the residual load to a common level over the whole 24-hour period. As the quadratic programming does not differ between positive and negative residual peaks due to the squaring, it would also try to tackle the negative peaks, created from the PV generation feed-in, and adjust them to the same level. This is prevented by the separation into critical and uncritical quarter-hour steps. Hence, these negative peaks are not tackled in this work.

Power balance and the component operation limits are taken into account with the following constraints:

$$
\begin{aligned}
& P_{n}^{\text {system }}=P_{n}^{\text {pv,f }}-P_{n}^{\text {battery }} \\
& P_{n}^{\text {battery }}=P_{n}^{\text {battery, charge }}+P_{n}^{\text {battery,discharge }}
\end{aligned}
$$




$$
\begin{aligned}
& P^{\text {battery,min }} \leq P_{n}^{\text {battery }} \leq P^{\text {battery,max }} \\
& S O C_{n}=S O C_{n-1}+\frac{P_{n-1}^{\text {battery, charge }} \cdot \eta^{\text {battery }} \cdot \Delta t}{C^{\text {battery }}}+\frac{P_{n-1}^{\text {battery }, \text { discharge }} \cdot \Delta t}{\eta^{\text {battery }} \cdot C^{\text {battery }}} \\
& S O C^{\text {min }} \leq S O C_{n} \leq S O C^{\text {max }}
\end{aligned}
$$$$
P_{n}^{\text {battery,charge }}=P_{n}^{\text {battery, }, \text { chargePV }}+P_{n}^{\text {battery, }, \text { chargeGrid }}
$$$$
P_{n}^{\text {battery, chargeGrid }} \geq 0
$$$$
0 \leq P_{n}^{\text {battery,charge } P V} \leq P_{n}^{p v, f}
$$

$$
0 \geq P_{n}^{\text {battery,discharge }} \begin{cases}\geq-P_{n}^{\text {demand }, f}+P_{n}^{p v, f}, & \text { if } P_{n}^{\text {demand }, f}>P_{n}^{p v, f} \\ =0, & \text { else }\end{cases}
$$

for all $\mathrm{n} \in \mathrm{N}$.

Eq. 2 specifies that the system output is the balance between the forecasted PV power generation $\left(P_{n}^{p v, f}\right)$ and

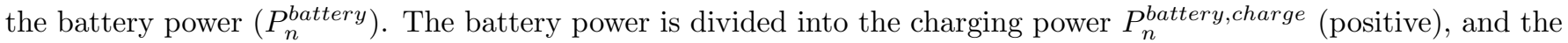

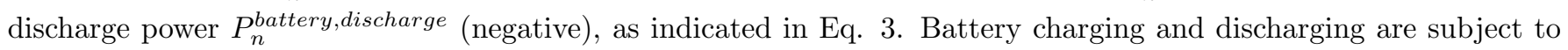
power limits (Eq. 4). Eq. 5 tracks the battery SOC, which must fall into its boundary values as shown in Eq. 6 . Eq. 7 specifies the power for battery charging, which is possible from the grid (Eq. 8) and from the available PV generation $P_{n}^{p v, f}$ (Eq. 9). Discharging into the grid is restrained by Eq. 10, as the discharge power is limited to the residual load (i.e., the difference between the forecasted load $P_{n}^{\text {demand,f }}$ and the PV generation $P_{n}^{p v, f}$ ).

The battery power is divided into charging and discharging power (Eq. 3). It is physically impossible to charge and discharge the battery simultaneously, but this circumstance might occur in mathematical models. In the literature, binary variables are used to avoid simultaneous charging and discharging in the same timestep, but are often accompanied by increased computational expenses. The first-stage optimization is implemented without such a strategy, because an optimal solution, which includes simultaneous charging and discharging, can always be rescheduled to avoid simultaneous charging and discharging, even without losing optimality. If the battery is charged at $P_{n}^{b a t t e r y, c h a r g e}$ and

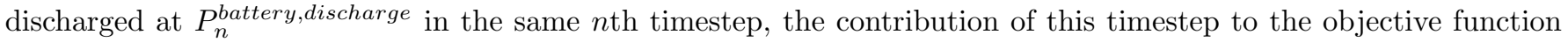
equals the case of having the battery charged at $P_{n}^{\text {battery,charge }}+P_{n}^{\text {battery,discharge }}$ if $P_{n}^{\text {battery,charge }} \geq-P_{n}^{\text {battery,discharge }}$ or discharged at $P_{n}^{\text {battery,discharge }}+P_{n}^{\text {battery, charge }}$ if $P_{n}^{\text {battery, charge }}<-P_{n}^{\text {battery,discharge }}$. Both cases, with and without simultaneous charging and discharging, result in the same objective function value in the $n$th timestep. In addition, the case without simultaneous charging and discharging activities, would have a higher SOC at the end of the $n$th 
timestep compared to the case with simultaneous charging and discharging, which might help to further support the objective in subsequent timesteps. The equivalent objective function value in the $n$th timestep and the higher SOC in the end of the $n$th timestep explain that without simultaneous charging and discharging, the solution will perform at least as well as with simultaneous charging and discharging.

\subsubsection{Threshold Relaxation}

The residual load profiles before and after the first-stage optimization are now the basis to derive the threshold power, which is applied in the second-stage optimization and the real-time battery dispatch later on. The residual load profile after optimization is analyzed regarding its highest peak demand during critical quarter-hour steps (cf. Section 3.1.1). This peak demand, further referred to as $P^{\text {threshold,minimal }}$, is the lowest achievable peak demand with the optimization. Setting a demand limit lower than $P^{\text {threshold,minimal }}$ would result in the infeasibility of the optimization. The residual load profile before the optimization is analyzed the same way to derive the upper bound of the demand limit $P^{\text {threshold,maximal }}$, as a higher value than $P^{\text {threshold,maximal }}$ would not be useful.

The first-stage optimization does not include the uncertainties of the system, which mainly come from deviations between the forecasted and actual profiles. Therefore, the lower bound $P^{\text {threshold,minimal }}$ is very optimistic. To adjust the demand limit, a relaxation factor $(\alpha)$ is used, which sets the demand limit between the upper and lower bound based on Eq. 11.

$$
P^{\text {threshold,initial }}=P^{\text {threshold,minimal }}+\alpha \cdot\left(P^{\text {threshold,maximal }}-P^{\text {threshold,minimal }}\right)
$$

Figure 5 illustrates the concept of threshold power relaxation. In this figure, the blue solid line represents the residual load profile before optimization while the red solid line represents the residual load profile after the optimization. Several values of the threshold power are marked as the dashed line with different relaxation factors $(\alpha=0$,

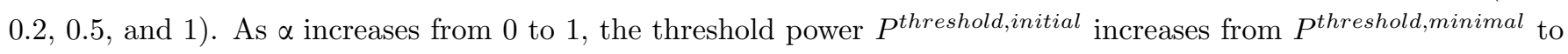
$P^{\text {threshold,maximal }}$. It can be seen in Figure 5 that higher peaks than $P^{\text {threshold,minimal }}$ may occur during uncritical quarter-hour steps (gray highlighted). These peaks in uncritical quarter-hour steps can be neglected, as they originate from grid charging, which will be addressed in the second-stage optimization to avoid higher loads than the threshold. During uncritical quarter-hour steps, the objective function value is not affected immediately regardless the PV-battery system's power flows. Due to the battery's ability to shift energy between the periods, these uncritical timesteps are still used to charge the battery, but the charging and discharging activities are less controlled compared to critical timesteps. Therefore, some of these activities may be deemed unnecessary from the perspective of prolonging the battery life, but this circumstance is not investigated further because it has no impact on the overall objective of the approach. 


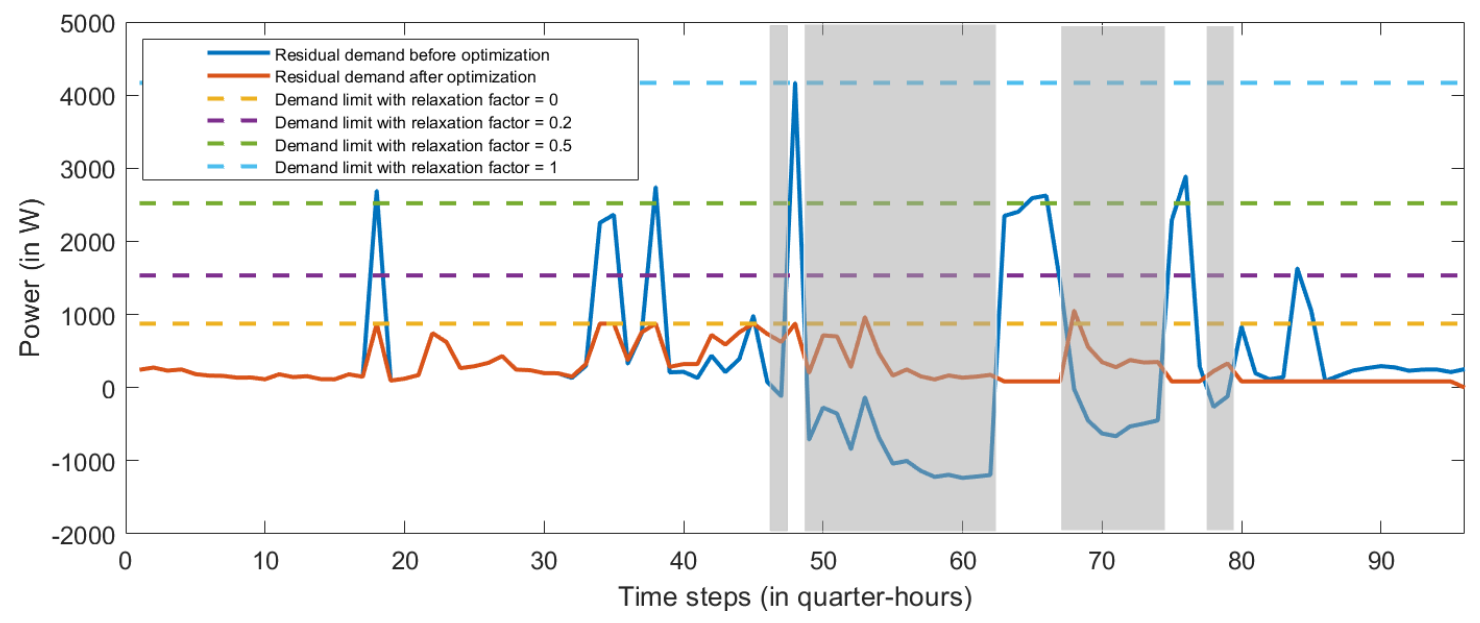

Figure 5: The threshold can be relaxed between the forecasted residual peak demand and residual peak demand after optimization (noncritical quarter-hour steps (gray highlighted) not considered). The dashed lines show different threshold powers depending on the relaxation factor of $\alpha=0,0.2,0.5$, and 1 .

\subsubsection{Second-stage Optimization: Battery Scheduling}

The second-stage optimization intends to determine the battery dispatch schedule. The threshold $P^{\text {threshold,initial }}$ from the first-stage optimization is used as the demand limit and is implemented into the optimization as a constraint (Eq. 12).

$$
P_{n}^{\text {demand,f }}-P_{n}^{\text {system }} \leq P^{\text {threshold }}
$$

The initial objective function becomes redundant with the threshold. Therefore, Eq. 13 is set as the new objective function. This objective function keeps the battery SOC at a high level and further reduces discharging activities to a minimum. To avoid charging and discharging at the same time, the battery discharging power $P_{k}^{\text {battery,discharge }}$ is included in the second-stage objective function. Minimizing the discharging activities (by maximizing $P_{k}^{\text {battery,discharge }} \leq 0$ ) avoids all unnecessary discharges, which includes those discharging activities, which would take place when charging and discharging occurred simultaneously with battery charging.

$$
\max f\left(S O C_{n}, P_{n}^{\text {battery, discharge }}\right)=\sum_{n=1}^{N}\left(S O C_{n}+\frac{P_{n}^{\text {battery }, \text { discharge }} \cdot \Delta t}{\eta^{\text {battery }} \cdot C^{\text {battery }}}\right)
$$

Though the charging from the grid is now limited to $P^{\text {threshold,initial }}$ with Eq. 12 (compare to Eq. 8), using it as the upper limit for grid charging might provide more leeway than in the first-stage optimization, as on one hand, the new objective function does not aim for the minimal demand limit and, on the other hand, the relaxed threshold could be higher than the lower bound of the demand limit $\left(P^{\text {threshold,initial }} \geq P^{\text {threshold,minimal }}\right)$. This additional leeway could result in high fluctuations, as the battery could be charged up to the limit of $P^{\text {threshold,initial }}$ at all time. Therefore, Eq. 14 is implemented. In the beginning of the day, $P_{1}^{\text {occurred }}$ is set as $P_{1}^{\text {occurred }}=0.5 \cdot P^{\text {threshold,initial }}$. Over the day, $P_{n}^{\text {occurred }}$ is increased, if a higher peak occurred, but is limited to $P^{\text {threshold,initial }}$. This constraint has one more advantage: If the actual residual peak load of a day is below $P^{\text {threshold,initial }}$ before optimization, that peak is not increased to $P^{\text {threshold,initial }}$ because of the grid charging option. 


$$
0 \leq P_{n}^{\text {battery,chargeGrid }} \leq \begin{cases}P_{n}^{\text {occurred }}, & \text { if } P_{n}^{\text {occurred }}<P^{\text {threshold,initial }} \\ P^{\text {threshold,initial }}, & \text { else }\end{cases}
$$

\subsection{Real-time Battery Dispatch by the Decentral Operator}

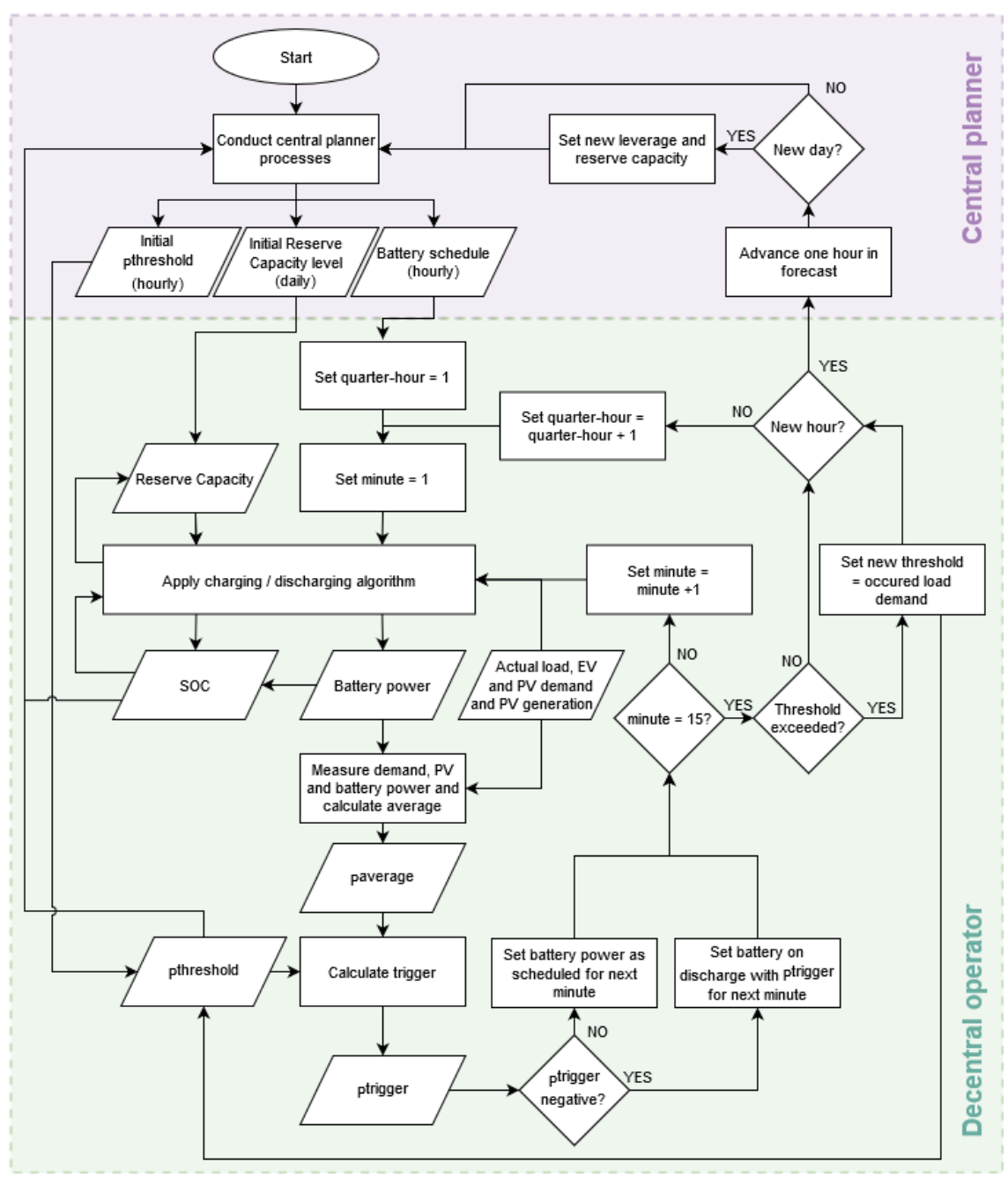

Figure 6: Approach from the decentral operator point of view.

The decentral operator implements the battery dispatch schedule sent by the central planner. The implementation process evolves on an hourly basis, depicted in Figure 6. The decentral operator manages battery charging and discharging in a 1-min time resolution, while the battery dispatch schedule specified by the central planner has a 15-min time resolution. Thus, within each 15-minute interval of the coming hour, the decentral operator starts with following the central planner's battery schedule first. However, actual PV generation and electric loads (i.e., building load and EV charging) may deviate from their forecasts. In cases of overestimated PV power or underestimated electric loads, strictly following the central planner's battery dispatch will unlikely maintain the predefined power limit $P^{\text {threshold }}$. Therefore, the trigger function from Hanna et al. (2014) is employed here. At the end of every minute, the residual load $P_{t}^{\text {average }}$ is calculated from the actual load and the PV generation over the elapsed period $T$, which includes the past and current minute steps. For example, after 3 minutes of the quarter-hour step, $\mathrm{T}=\{1$, $2,3\} . P_{t}^{\text {average }}$ is calculated as: 


$$
P_{t}^{\text {average }}=\frac{1}{t} \sum_{t=1}^{T}\left(P_{t}^{\text {load }}+P_{t}^{E V}-P_{t}^{P V \text { generation }}+P_{t}^{\text {battery }}\right), \forall t \in T
$$

The trigger function is then used to calculate the trigger power $P_{t}^{\text {trigger }}$ (Eq. 16).

$$
P_{t}^{\text {trigger }}=\frac{\left(P^{\text {threshold }}-P_{t}^{\text {average }}\right) \cdot 15}{15-t}
$$

Two possible cases can occur:

- $P_{t}^{\text {average }} \leq P^{\text {threshold}}$ : The trigger power is positive and the threshold is not jeopardized. In this case, the battery is charged and discharged according to the original dispatch schedule from the central planner.

- $P_{t}^{\text {average }}>P^{\text {threshold }}$ : The trigger power is negative and the threshold is jeopardized. In this case, the battery is discharged with $P_{t}^{\text {trigger }}$ in the next minute.

In Eq. 16, the denominator considers the elapsed time of the quarter-hour step. Thus, for the same magnitude of deviation, a later occurrence of having the average power exceeding the threshold power implies a higher value of the trigger power. During the application, the reduction of the peak can fail due to following reasons:

- The battery is empty $\left(S O C_{n}=S O C_{\min }\right)$ and no energy is available to meet the required discharge.

- $P_{t}^{\text {trigger }}<P^{\text {battery,min }}$, means that the trigger power is higher than the battery discharge power limit $\left(\left|P_{t}^{\text {trigger }}\right|>\right.$ $\left.\left|P^{\text {battery,min }}\right|\right)$, even though the battery has sufficient energy.

To support the real-time battery dispatch, reserve capacity $(\mathrm{RC})$ is introduced as another mechanism to tackle the forecasting uncertainties of electric load, PV power generation and EV charging. The RC can be understood as an analogy for operating reserve in power grid. Therefore, part of the battery capacity is reserved for emergency conditions when the power limit is jeopardized and unplanned battery discharge is needed. These emergency situations occur when the trigger power is negative and the free energy (energy not reserved) is not sufficient to cover the additional discharge.

The RC is only implemented in the real-time battery dispatch, not in the two-stage optimization. Recall that the first-stage optimization finds $P^{\text {threshold,minimal }}$. A constraint including the RC (i.e., $S O C^{\text {min }}+S O C^{\text {reserved }} \leq S O C_{n}$ $\leq S O C^{\max }$ instead of Eq. 6) would significantly narrow the bounds of the battery SOC and thereby generate a high threshold power. In addition, such a constraint would always cause infeasibility of the second-stage optimization as soon as the battery was discharged to a SOC level lower than $S O C^{\text {min }}+S O C^{\text {reserved }}$, which could occur frequently due to unexpected peaks. Therefore, it has been decided to remove the RC from the two-stage optimization, but consider it only in the real-time battery dispatch.

\subsection{Update Battery Scheduling}

After the decentral operator completes the real-time implementation of the battery dispatch for an entire hour with its four quarter-hour steps, the second-stage optimization is repeated by the central planner for the remaining hours of the 24-hour window. During this repetitive process, the second-stage optimization problem is updated from the following three aspects: First, the time horizon shrinks gradually from 24 hours to 1 hour. Second, the initial SOC $\left(S O C_{n-1}\right.$ in Eq. 5) is updated to the SOC level at the end of the preceding hour. And third, the threshold power $\left(P_{\text {threshold }}\right.$ in Eq. 11) may be updated if the battery dispatch fails in the preceding hour, as discussed earlier. 
With the exception of the above-mentioned updates, all other inputs to the second-stage optimization keep unchanged. Rerunning the second-stage optimization is necessary because of the deviation between the forecasted and actual data.

If all 24 hours are covered, the central planner repeats the first-stage optimization again with the corresponding $\alpha$ and RC. This process of staged optimization by the central planner and the real-time battery dispatch by the decentral operator is continued until the last hour of the considered time period is covered. The approach generates two main profiles, which are the basis of result analysis (cf. Hanna et al. (2014)). The first profile is the residual load profile of the actual load data before the battery operation, which is referred to as pre-Demand Charge Management (pre-DCM) profile (Eq. 17) and is calculated as following.

$$
P_{n}^{\text {pre-DCM }}=P_{n}^{\text {demand,actual }}-P_{n}^{\text {pv,actual }}, \forall n \in N
$$

The second profile, which includes the battery operation, is referred to as post-Demand Charge Management (post-DCM) profile (Eq. 18).

$$
P_{n}^{\text {post-DCM }}=P_{n}^{\text {demand,actual }}-P_{n}^{\text {pv,actual }}+P_{n}^{\text {battery }}, \forall n \in N
$$

\section{Input Data and Uncertainty Modeling}

This section describes the implemented PV-battery system of the decentral operator, the data and the methodology used to derive the day-ahead forecasts, and the methods to model forecasting uncertainty and to derive the relaxation factor $\alpha$ as well as the RC.

\subsection{Technical Parameters of the Case Study}

The underlying PV-battery system is implemented as a typical small residential system with a peak load of $2 \mathrm{~kW}$ for the PV system and a considerable battery size of $5 \mathrm{kWh}$. The EV can be charged at a rate of $3.5 \mathrm{~kW}$ and its fuel efficiency is assumed to be $17 \mathrm{kWh}$ per $\mathrm{km}$ (cf. Table 1).

\begin{tabular}{|l|l|}
\hline Parameters & Value \\
\hline Maximal charging power of the battery $P^{\text {battery,max }}$ & $5 \mathrm{~kW}$ \\
\hline Maximal discharging power of the battery $P^{\text {battery }, \text { min }}$ & $-5 \mathrm{~kW}$ \\
\hline Maximal state of charge of the battery $S O C^{\text {max }}$ & $90 \%$ \\
\hline Minimal state of charge of the battery $S O C^{\text {min }}$ & $10 \%$ \\
\hline Initial charge of the battery SOC & $50 \%$ \\
\hline Battery capacity $C^{\text {battery }}$ & $5 \mathrm{kWh}$ \\
\hline Charging and discharging efficiency of the battery $\eta^{\text {battery }}$ & $93 \%$ \\
\hline Charging the battery from the grid & Allowed \\
\hline Discharging the battery into the grid & Not allowed \\
\hline Installed PV capacity & $2 \mathrm{kWp}$ \\
\hline Charging power of the charging station & $3.5 \mathrm{~kW}$ \\
\hline Energy consumption of EV & $17 \mathrm{kWh} / 100 \mathrm{~km}$ \\
\hline
\end{tabular}

Table 1: All assumptions regarding PV-battery system. 


\subsection{Load and PV Data}

The electric load and PV generation profiles are provided by the German distribution system operator NetzeBW. The measured load and PV power have a one-minute resolution and cover a period of 13 months (cf. Figure 7). To derive the load forecast profile, the measured load data is first accumulated to a 15-minute resolution. Afterwards, the two electric load measurements observed at the same quarter-hour and same day of the week from the previous two weeks are averaged. The forecasts of PV power generation are based on a commercial forecast by a partner of NetzeBW but are normalized and scaled to the PV size used in this work. Both forecasts have a 15-min resolution and are depicted in Figure 8.

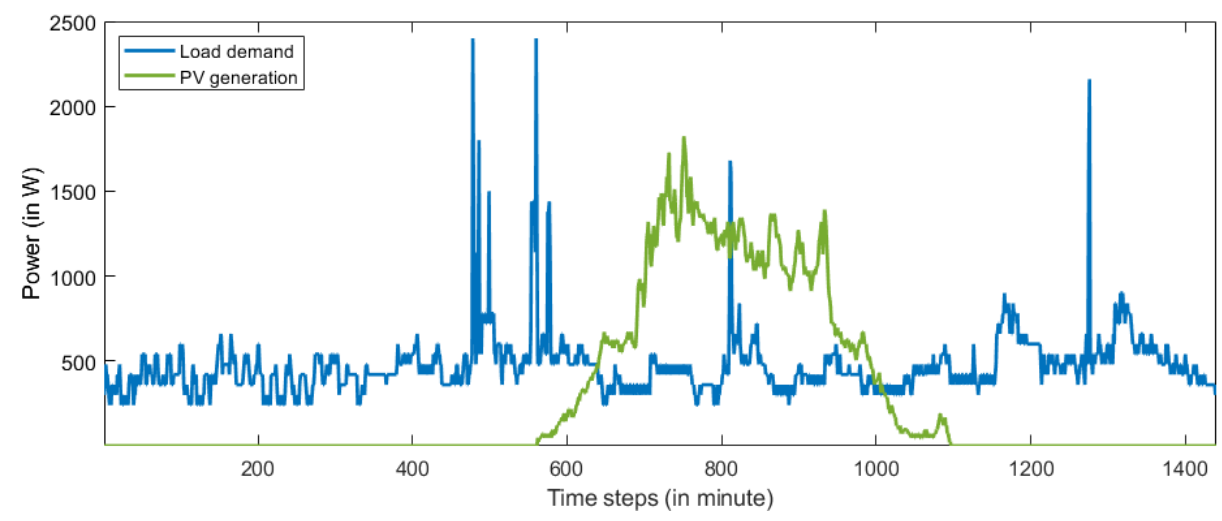

Figure 7: Example of smart meter measurement data for one day of the time series.

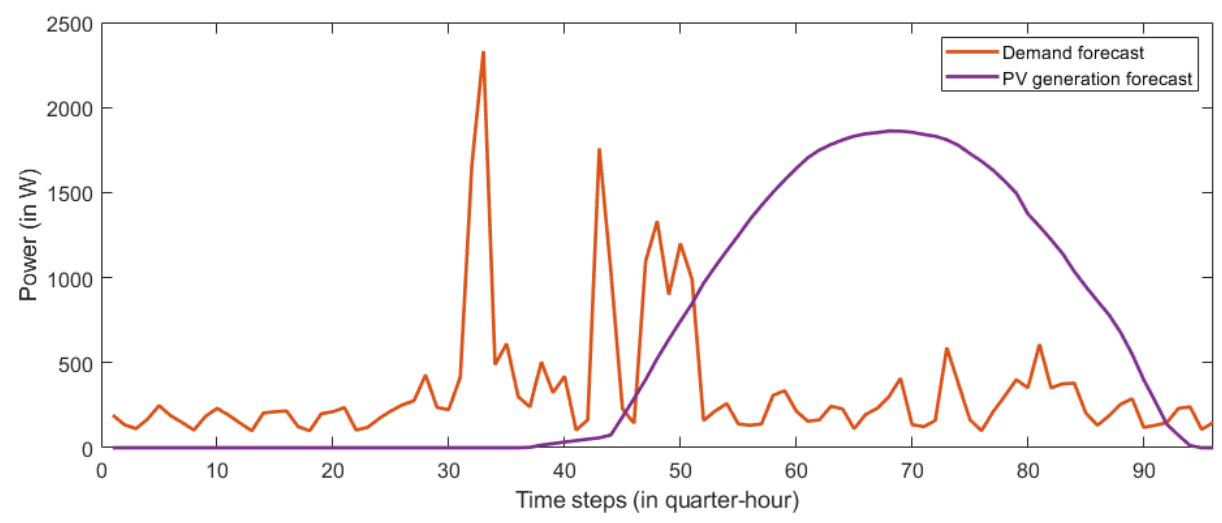

Figure 8: Corresponding load and PV generation forecast.

\subsection{EV Data}

The EV charging profile is based on the German Mobility Panel (Nobis and Kuhnimhof, 2018). The data includes all journeys of several thousand car owners over the period of one week. Every journey is characterized with the purposes, the driven distance and the departure and arrival time. The purpose of the journeys are divided into driving home, driving to work, driving to public places, and driving to other places. To derive the EV charging profile, one car owner of the data set is selected and its driving pattern is assumed to be the actual driving pattern during the week. It is further assumed that the owner charges every day when arriving at home. The energy demand is obtained from the driven distance over the day and the energy consumption of the EV. Combined with the assumed charging infrastructure and charging behavior of the EV, the charging profile is derived. The forecasted profile of the EV is generated similarly, but instead of using the data of one single owner, we use the average of several owners with similar 
driving patterns. As the data covers only one week, it is assumed that the driving patterns do not change over the year.

\subsection{Uncertainty Modeling}

The forecasting uncertainty is represented by probability distribution functions (PDFs), which are created for every quarter-hour of the day, every day type (Monday-Thursday, Friday, Saturday, Sunday) and every season (winter, summer, transition), resulting in a total of 1152 PDFs (96 quarter-hours $\mathrm{x} 3$ season types $\mathrm{x} 4$ day types). The deviations between forecasts and actual measurements are calculated and these deviations are pooled together for the same quarter-hour, the same day type, and the same season. A kernel distribution estimation is used to derive the PDFs for each pool.

The data of load, PV generation, and EV is not sufficient enough to cover all the iterations of the Monte Carlo simulation. Therefore, synthetic profiles are generated to model the actual measured profiles. These synthetic profiles are derived from the day-ahead forecast and the uncertainty (noise) $\xi_{n}^{\text {noise }}$, generated from the PDFs, for each n (Eq. 19). This approach resembles the one in Seddig et al. (2019).

$$
P_{n}^{\text {simulated }}=P_{n}^{\text {forecast }}-\xi_{n}^{\text {noise }}
$$

To model the whole season correctly, the forecast is always drawn randomly from a pool of all day-ahead forecasts of the season with the same day type.

This approach is used to create 1000 profiles and they are applied in the Monte Carlo simulation. All generated profiles have a 15 -minute resolution.

\subsection{Mechanisms to address Forecasting Uncertainty}

In Section 3.1.2, the relaxation of the threshold power via $\alpha$ was discussed, as well as the reserve capacity in Section 3.2. A Monte Carlo simulation is conducted to find appropriate values for both the RC and the $\alpha$. For every combination of day type (i.e., Monday-Thursday, Friday, Saturday, and Sunday) and season (i.e., winter, summer, and transition), the Monte Carlo simulation is performed with 1000 runs of the two-stage optimization and the battery operation (cf. Figures 4 and 6), one randomly selected day per run. Each day has its own synthetic profiles for electric load, PV power generation, and EV charging, which are generated according to the approaches discussed earlier in Section 4.4. Because all generated synthetic profiles have a 15-minute resolution, flat power consumption in each quarter-hour step is assumed to meet the one-minute resolution required by the real-time operation (cf. Figure 6).

From the results by the Monte Carlo simulation, the average peak demand reduction of all runs with the same combination of $\mathrm{RC}$ and relaxation factor is calculated using the following equation:

$$
P_{i, j}^{\text {difference,average }}=\frac{\sum_{k=1}^{K_{i, j}}\left(P_{i, j}^{\text {pre-DCM peak }}-P_{i, j}^{\text {post-DCM peak }}\right)}{K_{i, j}}
$$

where $P_{i, j}^{\text {difference,average }}$ is the average peak demand reduction resulting from the demand charge management for all one-day runs $K_{i, j}$ with the same RC (where $i$ is the share of the battery capacity) with $i \in[0 \%, 10 \%, \ldots, 70 \%, 80 \%]$, and the same relaxation factor $(j)$ with $j \in[0,0.1, \ldots, 0.9,1]$. Table 2 is a heat map table showing the results from one Monte Carlo simulation, with the optimal settings of RC and relaxation factor being $80 \%$ of the battery capacity and 0.2 , respectively. Certainly, the optimal setting may vary with different combinations of day type and season. 


\begin{tabular}{c|c|c|c|c|c|c|c|c|c|c|c|c}
\multicolumn{1}{c|}{} & 0 & 0.1 & 0.2 & 0.3 & 0.4 & 0.5 & 0.6 & 0.7 & 0.8 & 0.9 & 1 \\
\hline $0 \%$ & 742 & 623 & 585 & 678 & 702 & 654 & 644 & 672 & 643 & 392 & 500 \\
$10 \%$ & 682 & 665 & 924 & 954 & 883 & 859 & 741 & 725 & 477 & 464 & 486 \\
$20 \%$ & 579 & 938 & 889 & 884 & 959 & 824 & 640 & 751 & 632 & 602 & 598 \\
$\mathrm{RC}$ & $40 \%$ & 929 & 886 & 902 & 1097 & 1147 & 917 & 701 & 852 & 738 & 590 & 650 \\
& 1032 & 958 & 1315 & 1230 & 1121 & 1080 & 838 & 934 & 748 & 593 & 576 \\
& $50 \%$ & 1263 & 998 & 1061 & 1297 & 1182 & 1156 & 1086 & 913 & 679 & 569 & 425 \\
$60 \%$ & 1020 & 1245 & 1353 & 1352 & 1077 & 1330 & 935 & 1085 & 809 & 707 & 558 \\
$70 \%$ & 1037 & 1183 & 1397 & 1381 & 1126 & 927 & 971 & 889 & 813 & 624 & 451 \\
$80 \%$ & 913 & 1251 & 1423 & 1222 & 1164 & 917 & 933 & 726 & 702 & 597 & 693
\end{tabular}

Table 2: Average difference between pre-DCM and post-DCM peak demand for every combination of relaxation factor and reserve capacity for the weekday samples.

\subsection{Computing Environment and Time Complexity}

The two-stage optimization model, battery real-time dispatch, and uncertainty modeling are all implemented in Matlab, a numerical computing environment and programming language. To assure that the global minimum is achieved, the global search algorithm of Ugray et al. (2007), which uses several starting points and the corresponding results to estimate the basin radius and thresholds to find the global minimum, is applied.

The computational expense is largely caused by the Monte Carlo simulation, which simulates the two-stage optimization and the unit commitment of the battery schedule. The application of the quadratic optimization is NP-hard (Pardalos and Vavasis, 1991), but the time complexity is mainly driven by the number of samples in the Monte Carlo simulation and increases linear with these. Therefore, the time complexity of the approach is assumed to be $O(n)$. This computation expenses are borne by the central planner, who applies the Monte Carlo simulation and the twostage optimization. The application of the battery schedule, the power measurements, the calculations of the trigger function and the rescheduling of the battery due to the $\mathrm{RC}$ limitation on the side of the decentral operator for one hour are conducted in the simulation in less than one second. Hence, the computational effort seems to be applicable for limited calculation capacities of the decentral operator.

\section{Results and Discussion}

\subsection{Post-DCM Residual Electric Load}

The pre- and post-DCM residual load profiles are compared in Figure 9. The daily peak demands (pre- and postDCM) and their differences are summarized in Table 3, which shows that the peak demand has been reduced for all seven days. The daily peak demand reduction ranges from $557 \mathrm{~W}$ on Day 7 (Saturday) to $2685 \mathrm{~W}$ on Day 2 (Monday). Percentage-wise, the post-DCM peak demand is reduced from the pre-DCM peak by $17 \%$ on Day 7 and $52 \%$ on Day 2. For the purpose of reference, Table 4 provides the values of RC and $\alpha$ derived from the Monte Carlo simulation and the initial threshold power obtained from the first-stage optimization at the beginning of each day. By comparing the post-DCM peak in Table 3 and the initial threshold power in Table 4, we can see that the initial threshold power has been successfully maintained for most days (Days 2, 3, 4, 5 and 7). Day 1 and day 6 show slight deviations between initial threshold and achieved reduction. 


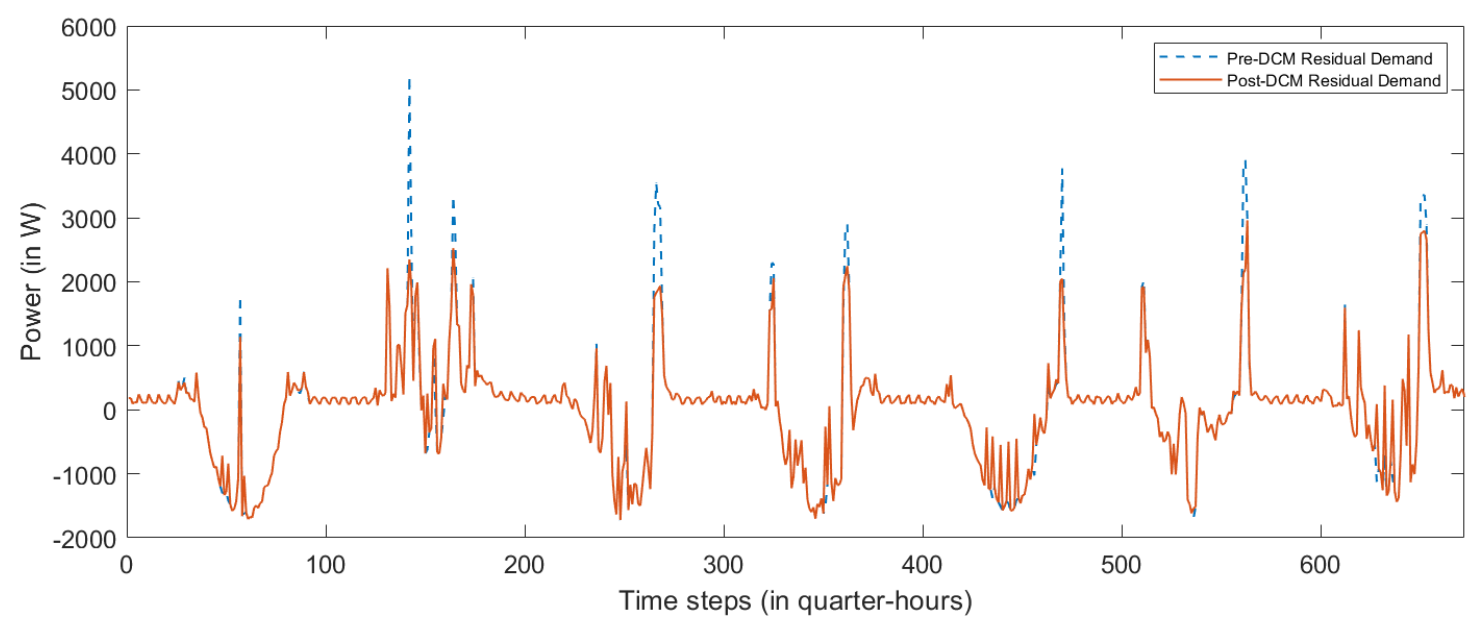

Figure 9: Pre-DCM residual load (blue dashed) and post-DCM residual load (red) over the whole simulation period.

\begin{tabular}{l|l|l|l|l|l|l|l} 
& Day 1 & Day 2 & Day 3 & Day 4 & Day 5 & Day 6 & Day 7 \\
\hline Pre-DCM peak [W] & 1780 & 5212 & 3547 & 2898 & 3773 & 3901 & 3355 \\
Post-DCM peak [W] & 1140 & 2527 & 1936 & 2247 & 2014 & 2962 & 2798 \\
\hline Difference [W] & 640 & 2685 & 1611 & 651 & 1719 & 939 & 557 \\
Reduction [\%] & $36 \%$ & $52 \%$ & $45 \%$ & $22 \%$ & $46 \%$ & $24 \%$ & $17 \%$
\end{tabular}

Table 3: Pre-DCM peak, post-DCM peak and difference for every day of the simulation in Watt starting with day 1 (Sunday).

\begin{tabular}{l|l|l|l|l|l|l|l} 
& Day 1 & Day 2 & Day 3 & Day 4 & Day 5 & Day 6 & Day 7 \\
\hline Reserve Capacity [\%] & 40 & 80 & 80 & 80 & 80 & 0 & 60 \\
Relaxation Factor & 1 & 0.2 & 0.2 & 0.2 & 0.2 & 0.8 & 0.8 \\
Initial Threshold [W] & 1170 & 2527 & 1936 & 2248 & 2049 & 2876 & 2798
\end{tabular}

Table 4: Reserve capacity, relaxation factor and initial threshold for the simulated period.

\subsection{Comparison of Different Approach Settings}

The advantages of considering forecasting uncertainties for peak demand reduction are demonstrated further by comparing the approach presented in this paper with other approaches or different approach settings (scenarios). The different scenarios are:

- Scenario 1: The approach exactly as presented in this paper (yellow solid line in Figure 10).

- Scenario 2: The approach presented in this paper, but not using the $\alpha$ and RC, i.e. $\alpha=0$ and $\mathrm{RC}=0$ (purple solid line in Figure 10).

- Scenario 3: The approach exactly as presented in this paper, but assuming a perfect forecast (red solid line in Figure 10). In this scenario, $\alpha$ and $\mathrm{RC}$ are not needed, as no uncertainties exist.

- Scenario 4: A simple battery management approach, which charges the battery whenever the PV power is more than the total electric loads (building and EV) and discharges the battery whenever the PV power is less than the total electric loads (green dotted line in Figure 10). 


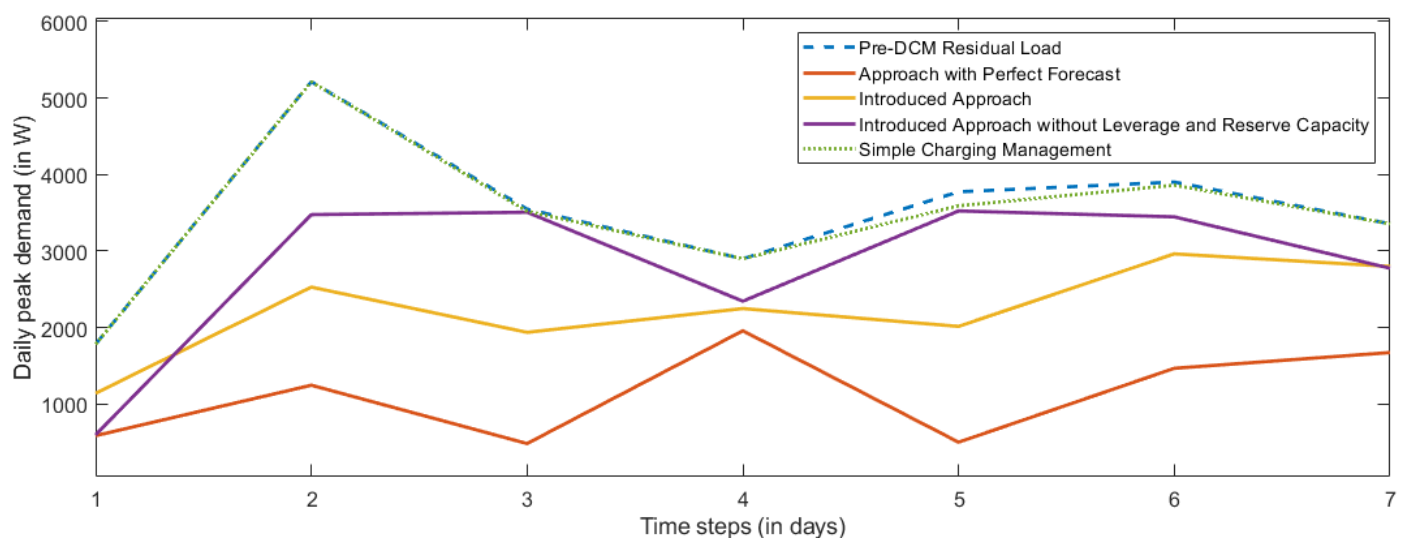

Figure 10: Comparison of the highest daily post-DCM residual load peak of the different scenarios. It is visible that the perfect forecast Scenario (red) performs best, followed be the approach presented in this work (yellow). Without using the RC and $\alpha$ (Scenario 2 (purple)), the approach does not perform well and consistently. Simpler battery management approaches (Scenario 4 (green dotted)) are not suited for peak shaving applications.

These different scenarios are simulated for the same week as presented in Section 5.1 and the post-DCM daily peak for all scenarios are depicted in Figure 10. It is visible, that Scenario 3 has the lowest daily peak demand and therefore, performs best. This is anticipated, as the approach uses perfect forecast and the demand limit in the first-stage optimization can be hold during the battery dispatch. Without the mechanisms to tackle the uncertainty, Scenario 2 does not perform well and consistently. The peaks are close to or almost as high as to the pre-DCM daily peaks for most days. The relaxation factor $\alpha$ on this day could be lower to further reduce the peak demand. The simple charging and discharging management (Scenario 4) does not reduce the peak demand at all and, therefore, seems to be unsuitable for such an application.

\subsection{Impact of Relaxation Factor and Reserve Capacity on the post-DCM Profile}

To investigate the impact of $\alpha$ and RC on peak demand reduction, Scenario 1 and Scenario 2 from Section 5.2 are compared to each other with respect to the threshold power, the SOC profile, and the residual load profile. The preDCM residual load profile is seen in Figure 11a. In addition, the initial threshold power is depicted for both scenarios. As Scenario 2 has $\alpha$ of 0 during all days, the threshold is also lower than in Scenario 1. Based on these thresholds, the battery schedule is planned and operated. Figure 11b shows the impact on the SOC level of both scenarios during the considered period. Scenario 1 has a RC of $40 \%$ on the first day, which keeps the SOC level in the beginning of the period on a constant level, as no discharge without peak is allowed below this level. A discharge of the battery is only conducted for the single peak during this day. In comparison, the SOC profile of Scenario 2 shows an immediate drop in the beginning of the period even without a peak demand because the threshold power is set to almost $0 \mathrm{~W}$ (such a low threshold is only possible as the battery was implemented with $\mathrm{SOC}=50 \%$ in the beginning). This way, even the low loads will be reduced, depleting the battery fast. Over the whole period, the battery in Scenario 2 is discharged to $S O C^{\text {min }}$ ten times, in contrast with three times in Scenario 1. Every time the battery is discharged, the post-DCM residual load jumps back to the pre-DCM residual load, which is visible in Figure 11c. This is especially the case for Scenario 2 on day $2,3,5$ and 6. 


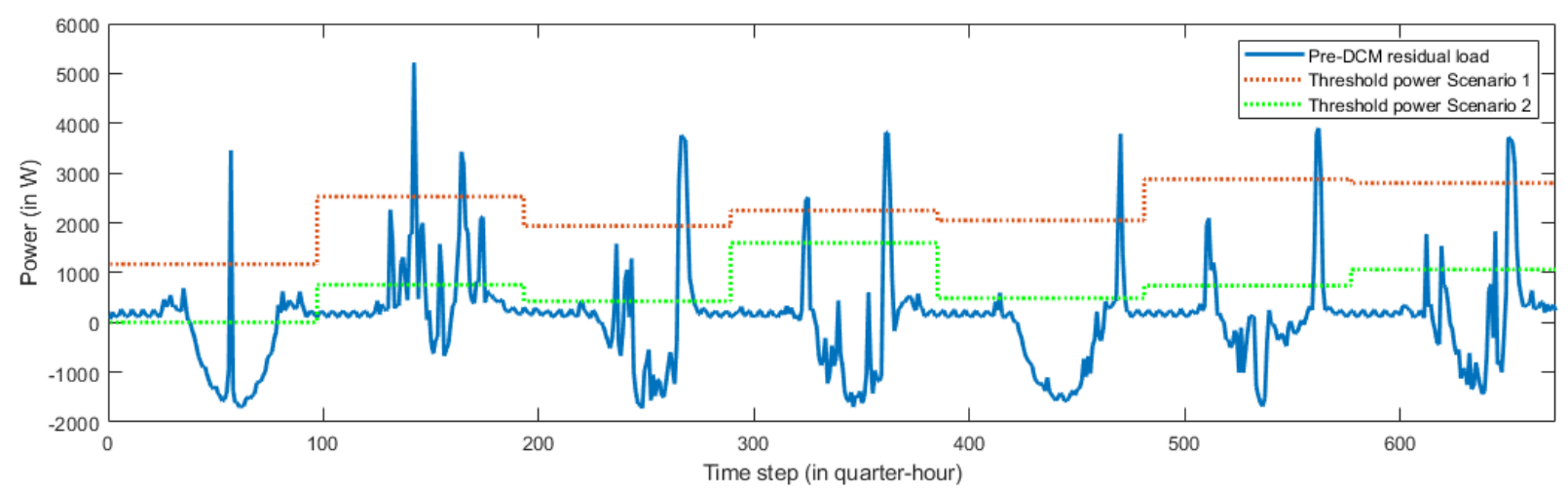

(a) Pre-DCM electric load and thresholds based on the relaxation factors for Scenario 1 (red) and Scenario 2 (green).

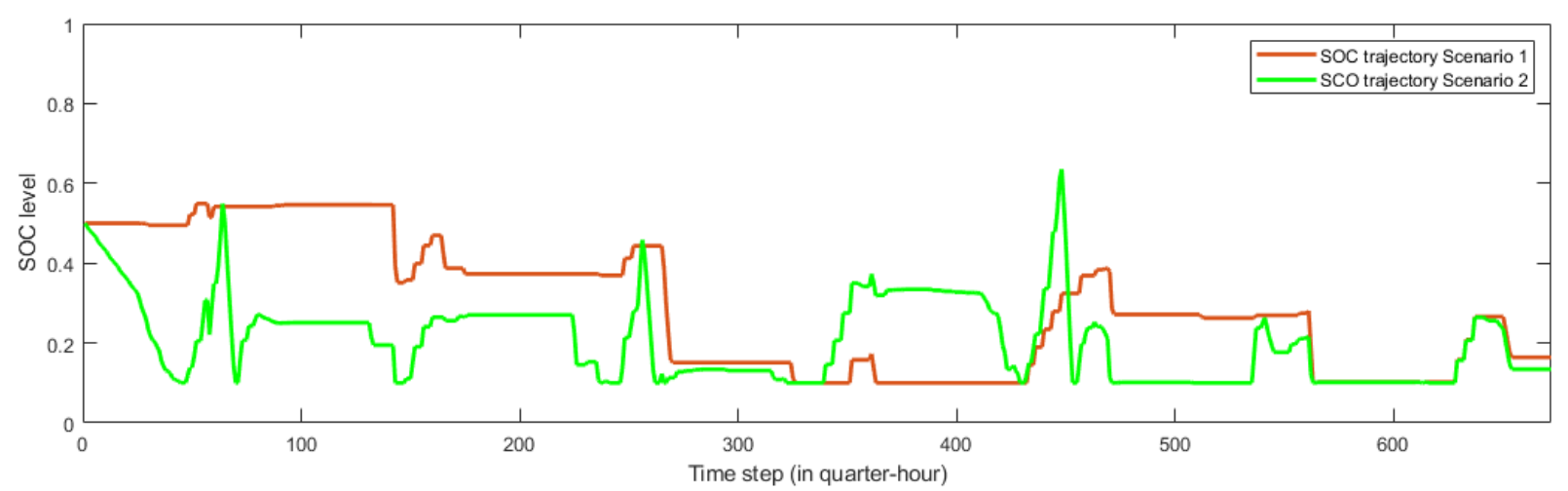

(b) Corresponding SOC trajectory of Scenario 1 (red) and Scenario 2 (green).

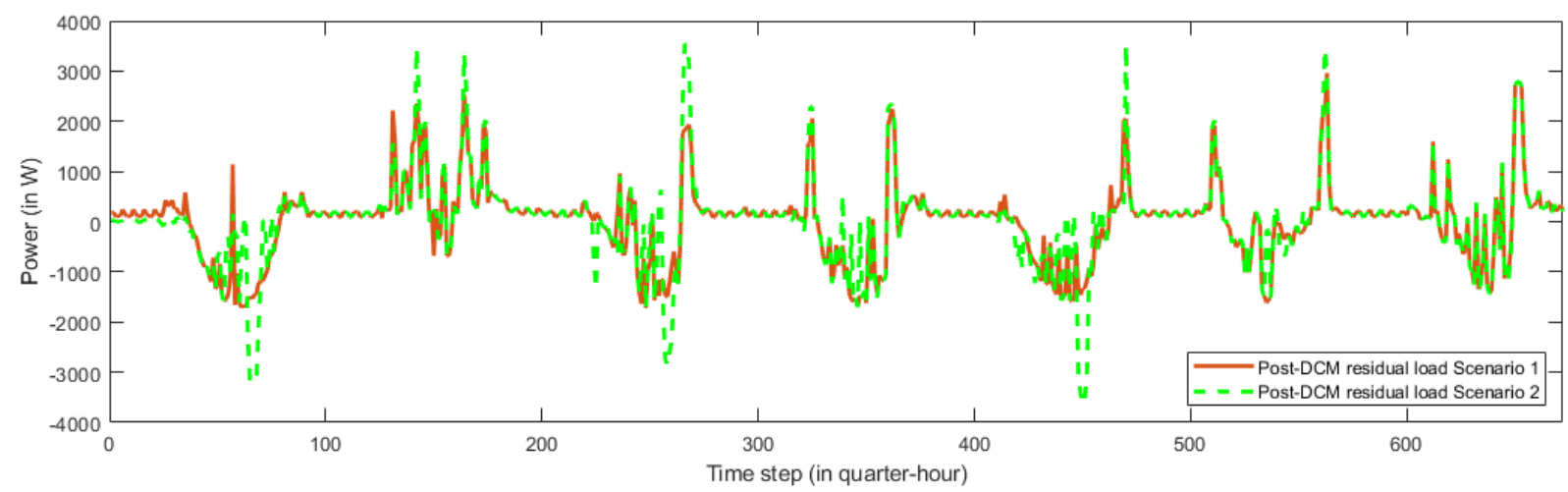

(c) Exemplary post-DCM residual load of Scenario 1 (red) and Scenario 2 (green dashed).

Figure 11: Pre-DCM residual load with threshold of different scenarios (a), resulting SOC trajectory of scenarios (b) and residual load post-DCM of scenarios (c). 


\section{Conclusions and Outlook}

Reliable, local control of load flexibilities is a key competence of future system operators due to the increasing share of renewable energy generation and a potential high penetration of electric vehicles. The proposed approach delivers a reliable solution to set the threshold power and to constrain the power consumption below that limit using the flexibility of distributed electrical storage. The advantages of the approach have been demonstrated through a case study, which achieved daily peak demand reduction by $17 \%$ to $52 \%$ for one week. The novelty of the approach lies in the following aspects:

- Current approaches in literature often assume a perfect insight into the decentral operator's photovoltaic-battery system without considering privacy issues. Our approach supports the central planner's objectives without sharing load profiles. Only the decentral operator's information on its system, the total annual energy consumption and the process tracking information are used by the central planner to schedule the decentral operator's battery.

- In addition, such literature does not wonder about the computational expenses and transfers these to the decentral operators without considering the available infrastructure. The proposed approach of this work supports the application on systems with limited calculation capacities at decentral operators, whose task is simply to apply the battery schedule.

- The consideration of uncertainties is a recent development in the literature on photovoltaic-battery systems, but often focuses only on load demand and photovoltaic generation. Electric vehicle uncertainties still lack consideration, even though their share rapidly increases and affects decentral distribution networks. Our approach considers these uncertainties and proposes two mechanisms, the relaxation factor and the reserve capacity, to address the underlying uncertainty of photovoltaic-battery systems caused by the deviations between the forecasted and actual profiles of load demand, electric vehicle demand and power generation. The relaxation factor implements an ambitious but realistic limit of the decentral operator's demand. The reserve capacity restrains the use of a certain share of the battery energy unless the power limit is jeopardized.

Though the approach is able to significantly reduce the daily peak load, there are several avenues worthwhile for future research. First, the second-stage optimization could be used for other objectives (e.g., minimization of the utility bill or maximization of battery life) while the threshold is implemented as a constraint. This paper focuses on peak demand shaving for a single household, the impact of which on the distribution grid is negligible. Therefore, one major improvement is to manage many residential households on a distribution feeder or district substation together, which would potentially leverage the diversity of load profiles for the reduction of aggregated power demand. In addition, further research on the impact of the electric vehicle on the photovoltaic-battery system is particularly interesting. Intelligent or bi-directional charging management could support the peak demand shaving further.

\section{Acknowledgement}

We want to thank the Reinhard Frank-Stiftung for financing the exchange program, where this work arose out of. We want to further thank the Energy Production and Infrastructure Center of the University of North Carolina at Charlotte, especially Christina Kopitopoulou, for their invitation and support. Last, thanks to the the German DSO NetzeBW for the provision of the data.

\section{References}

Alanne, K., Saari, A., 2006. Distributed energy generation and sustainable development. Renewable and Sustainable Energy Reviews 10, 539-558. doi:10.1016/j.rser.2004.11.004. 
Appino, R.R., González Ordiano, J.Á., Mikut, R., Faulwasser, T., Hagenmeyer, V., 2018. On the use of probabilistic forecasts in scheduling of renewable energy sources coupled to storages. Applied Energy 210, 1207-1218. doi:10.1016/j.apenergy.2017.08.133.

Arcos-Aviles, D., Pascual, J., Guinjoan, F., Marroyo, L., Sanchis, P., Marietta, M.P., 2017. Low complexity energy management strategy for grid profile smoothing of a residential grid-connected microgrid using generation and demand forecasting. Applied Energy 205, 69-84. doi:10.1016/j.apenergy.2017.07.123.

Buchmann, E., Kessler, S., Jochem, P., Böhm, K., 2013. The costs of privacy in local energy markets, in: 2013 IEEE 15th Conference on Business Informatics, IEEE. pp. 198-207.

Castillo-Cagigal, M., Caamaño-Martín, E., Matallanas, E., Masa-Bote, D., Gutiérrez, A., Monasterio-Huelin, F., Jiménez-Leube, J., 2011. Pv self-consumption optimization with storage and active dsm for the residential sector. Solar Energy 85, 2338-2348. doi:10.1016/j.solener.2011.06.028.

Chua, K.H., Lim, Y.S., Morris, S., 2015. Cost-benefit assessment of energy storage for utility and customers: A case study in malaysia. Energy Conversion and Management 106, 1071-1081. doi:10.1016/j.enconman.2015.10.041.

Correa-Florez, C.A., Gerossier, A., Michiorri, A., Kariniotakis, G., 2018. Stochastic operation of home energy management systems including battery cycling. Applied Energy 225, 1205-1218. doi:10.1016/j.apenergy.2018.04.130.

Dengiz, T., Jochem, P., 2020. Decentralized optimization approaches for using the load flexibility of electric heating devices. Energy 193, 116651.

Di Giorgio, A., Pimpinella, L., 2012. An event driven smart home controller enabling consumer economic saving and automated demand side management. Applied Energy 96, 92-103. doi:10.1016/j.apenergy.2012.02.024.

Erdinc, O., 2014. Economic impacts of small-scale own generating and storage units, and electric vehicles under different demand response strategies for smart households. Applied Energy 126, 142-150. doi:10.1016/j.apenergy.2014.04.010.

Farah, S., Whaley, D., Saman, W., 2016. Control strategies of domestic electrical storage for reducing electricity peak demand and life cycle cost. International Journal of Hydrogen Energy 41, 20939-20949. doi:10.1016/j.ijhydene.2016.06.164.

Hanna, R., Kleissl, J., Nottrott, A., Ferry, M., 2014. Energy dispatch schedule optimization for demand charge reduction using a photovoltaic-battery storage system with solar forecasting. Solar Energy 103, 269-287. doi:10.1016/j.solener.2014.02.020.

Hemmati, R., Saboori, H., 2017. Stochastic optimal battery storage sizing and scheduling in home energy management systems equipped with solar photovoltaic panels. Energy and Buildings 152, 290-300. doi:10.1016/j.enbuild.2017.07.043.

Jochem, P., Schönfelder, M., Fichtner, W., 2015. An efficient two-stage algorithm for decentralized scheduling of micro-chp units. European Journal of Operational Research 245, 862-874.

Kaschub, T., Jochem, P., Fichtner, W., 2016. Solar energy storage in german households: profitability, load changes and flexibility. Energy Policy 98, 520-532. doi:10.1016/j.enpol.2016.09.017.

Klingler, A.L., Teichtmann, L., 2017. Impacts of a forecast-based operation strategy for grid-connected pv storage systems on profitability and the energy system. Solar Energy 158, 861-868. doi:10.1016/j.solener.2017.10.052. 
Li, Y., Gao, W., Ruan, Y., 2018. Performance investigation of grid-connected residential pv-battery system focusing on enhancing self-consumption and peak shaving in kyushu, japan. Renewable Energy 127, 514-523. doi:10.1016/j.renene.2018.04.074.

Lopes, R.A., Martins, J., Aelenei, D., Lima, C.P., 2016. A cooperative net zero energy community to improve load matching. Renewable Energy 93, 1-13. doi:10.1016/j.renene.2016.02.044.

Lu, X., Zhou, K., Yang, S., Liu, H., 2018. Multi-objective optimal load dispatch of microgrid with stochastic access of electric vehicles. Journal of Cleaner Production 195, 187-199. doi:10.1016/j.jclepro.2018.05.190.

Luthander, R., Widén, J., Munkhammar, J., Lingfors, D., 2016. Self-consumption enhancement and peak shaving of residential photovoltaics using storage and curtailment. Energy 112, 221-231. doi:10.1016/j.energy.2016.06.039.

Matallanas, E., Castillo-Cagigal, M., Gutiérrez, A., Monasterio-Huelin, F., Caamaño-Martín, E., Masa, D., JiménezLeube, J., 2012. Neural network controller for active demand-side management with pv energy in the residential sector. Applied Energy 91, 90-97. doi:10.1016/j.apenergy.2011.09.004.

Nobis, C., Kuhnimhof, T., 2018. Mobilität in deutschland - mid ergebnisbericht: Studie von infas, dlr, ivt und infas 360 im auftrag des bundesministers für verkehr und digitale infrastruktur. URL: www mobilitaet-in-deutschland.de.

Pardalos, P.M., Vavasis, S.A., 1991. Quadratic programming with one negative eigenvalue is np-hard. Journal of Global Optimization 1, 15-22. doi:10.1007/BF00120662.

Paterakis, N.G., Erdinc, O., Pappi, I.N., Bakirtzis, A.G., Catalao, J.P.S., 2016. Coordinated operation of a neighborhood of smart households comprising electric vehicles, energy storage and distributed generation. IEEE Transactions on Smart Grid 7, 2736-2747. doi:10.1109/TSG.2015.2512501.

Ranaweera, I., Midtgård, O.M., 2016. Optimization of operational cost for a grid-supporting pv system with battery storage. Renewable Energy 88, 262-272. doi:10.1016/j.renene.2015.11.044.

Ratnam, E.L., Weller, S.R., Kellett, C.M., 2015. An optimization-based approach to scheduling residential battery storage with solar pv: Assessing customer benefit. Renewable Energy 75, 123-134. doi:10.1016/j.renene.2014.09.008.

Reihani, E., Motalleb, M., Ghorbani, R., Saad Saoud, L., 2016. Load peak shaving and power smoothing of a distribution grid with high renewable energy penetration. Renewable Energy 86, 1372-1379. doi:10.1016/j.renene.2015.09.050.

Riffonneau, Y., Bacha, S., Barruel, F., Ploix, S., 2011. Optimal power flow management for grid connected pv systems with batteries. IEEE Transactions on Sustainable Energy 2, 309-320. doi:10.1109/TSTE.2011.2114901.

Schwarz, H., Schermeyer, H., Bertsch, V., Fichtner, W., 2018. Self-consumption through power-to-heat and storage for enhanced pv integration in decentralised energy systems. Solar Energy 163, 150-161. doi:10.1016/j.solener.2018.01.076.

Seddig, K., Jochem, P., Fichtner, W., 2019. Two-stage stochastic optimization for cost-minimal charging of electric vehicles at public charging stations with photovoltaics. Applied energy 242, 769-781.

Thomas, D., Deblecker, O., Ioakimidis, C.S., 2018. Optimal operation of an energy management system for a gridconnected smart building considering photovoltaics' uncertainty and stochastic electric vehicles' driving schedule. Applied Energy 210, 1188-1206. doi:10.1016/j.apenergy.2017.07.035.

Ugray, Z., Lasdon, L., Plummer, J., Glover, F., Kelly, J., Martí, R., 2007. Scatter search and local nlp solvers: A multistart framework for global optimization. INFORMS Journal on Computing 19, 328-340. doi:10.1287/ijoc.1060.0175. 
Wang, Y., Lin, X., Pedram, M., 2014. Adaptive control for energy storage systems in households with photovoltaic modules. IEEE Transactions on Smart Grid 5, 992-1001. doi:10.1109/TSG.2013.2292518.

Wang, Z., Jochem, P., Fichtner, W., 2020. A scenario-based stochastic optimization model for charging scheduling of electric vehicles under uncertainties of vehicle availability and charging demand. Journal of Cleaner Production 254,119886 .

Zhong, Q., Khalilpour, R., Vassallo, A., Sun, Y., 2016. A logic-based geometrical model for the next day operation of pv-battery systems. Journal of Energy Storage 7, 181-194. doi:10.1016/j.est.2016.06.008. 